\title{
Feasibility of tracking laparoscopic instruments in a box trainer using a Leap Motion Controller
}

\author{
I. Oropesa ，T.L. de Jong ，P. Sánchez-González ， J. Dankelman ，E.J. Gómez
}

\begin{abstract}
A B S T R A C T
Motion analysis is employed to assess minimally invasive surgical psychomotor skills in box trainers. Tracking of laparoscopic instruments requires sensor-based systems that can be expensive, limit movements and modify their ergonomic properties. We evaluate the feasibility of using Leap Motion as a cheap, unobtrusive alternative. Four experiments were performed to determine its precision while tracking a laparoscopic instrument inside and outside a box trainer. Static long and short term precision of the Leap Motion was $<2.5 \mathrm{~mm}$. Precision between 12 different positions within the box trainer was $<0.7 \mathrm{~mm}$ for all measured distances between neighbors. Dynamic precision when moving the instrument for $200 \mathrm{~mm}$ ranged between 2 and $15 \mathrm{~mm}$. Leap Motion presents acceptable precision values inside a box trainer. Improvements are still required (e.g.: multiple instruments' tracking). Once solved, a validation study should verify the usefulness of Leap Motion to objectively measure skills of novices and residents during training.
\end{abstract}

\section{Introduction}

Minimally invasive surgery (MIS) interventions are performed through small incisions in the patient's body, as opposed to conventional surgery [1]. MIS has become a standard procedural routine in many surgical subspecialties; including laparoscopic, gynecologic, nephritic and colorectal surgery [1]. In the case of laparoscopy, within the abdominal cavity a pneumoperitoneum is generated by infusing $\mathrm{CO}_{2}$ gas, creating the working space for the surgeon. Long-shafted instruments are introduced through the incisions via special cannulas (trocars), and the patient's anatomy is recorded by means of an intra-corporeal endoscope and visualized on an external monitor [2].

Laparoscopic surgery is nowadays the preferred treatment method for a variety of procedures [3]. It is considered to be less painful and scarring to the patient, to reduce post-operative complications, decrease morbidity and mortality, and shorten hospital stays, thus reducing associated costs for clinical centers. However, it also introduces difficulties into the medical field, as it is technically demanding and requires different technical skills to those of open surgery [4].

Training and assessment of technical skills has become a major concern in MIS learning programs, especially 
considering the social demand for better-prepared professionals and for the decrease of medical errors [5]. Much effort is being put in the definition of structured learning programs, where practice with real patients in the operating room $(\mathrm{OR})$ can be delayed until the resident can attest for a minimum level of psychomotor competence [5].

Several training methods have been developed to increase the skills of novices and residents in patient-free environments. One of the most employed is that of the laparoscopic box trainer. The box offers an enclosed space simulating the surgical scenario, with realistic dimensions, trocar access points and camera view. Real laparoscopic instruments are employed to perform simple tasks inside these low cost surgical simulators. These tasks are usually simple abstractions of reality, offering reproducible and repeatable routines with the sole purpose of training/ assessing individual skills. It has been shown that these simulators do increase the skills of novices [6,7] and that those skills are transferable to the OR [8].

Finding the most adequate parameters to objectively evaluate basic laparoscopic skills is important. Studies have shown that objective measurements of laparoscopic skills include errors, time, and motion analysis of the laparoscopic instruments $[4,9]$. In particular, motion analysis has been extensively covered in the literature as a means of measuring performance differences between novices, residents and experts. Several authors have proposed scoring systems based on the movements of the laparoscopic instruments during basic training and assessment tasks, such as path length, average speed, or motion smoothness $[4,10]$. Reports have shown that in general these motion analysis metrics present good construct validity applied to technical skills' assessment, and nowadays their use is widely extended in commercial simulation systems $[9,11]$. Other authors have focused on the use of motion analysis to break down complex surgeries into a series of simplified tasks and maneuvers [12-15], combined with machine learning techniques such as hidden Markov models [12] or linear discriminant analysis [13] to automatically determine the steps in an intervention and assess skills in unstructured surgical tasks. The idea behind this approach is that in real surgical settings, the information provided by aggregated motion analysis metrics such as path length present a limited instructive value and do not describe tool motion sufficiently to provide formative feedback to trainees [14].

Motion analysis requires being able to track the movements of the laparoscopic instruments during performance of a task. Tracking technologies have traditionally relied on sensor-based systems, based on optical [16], electromagnetic [17] or mechanic [12] technologies. Nowadays, they are incorporated into different surgical systems for intraoperative navigation [18], robotic surgery [13] and surgical training (box trainers, virtual reality simulators) $[9,11]$. However, it can be argued that their use can modify the ergonomics and constrain movements of the instruments, altering the users' experience and performance. More recently, endoscopic video analysis is being explored as a means to provide tracking of instruments [19]. This alternative based on computer vision offers non-expensive and non-obtrusive tracking of laparoscopic instruments without the need of mounting sensors in them that may modify their ergonomics. However, the technology is still being perfected and is used mainly for research purposes, and is currently not commercially available.

Following the philosophy of providing affordable and unobtrusive computer vision-based alternatives, this study proposes the use of the Leap Motion Controller ${ }^{\text {TM }}$ (Leap Motion, Inc., San Francisco, CA, USA) as a tracking device for motion analysis during laparoscopic training in a box trainer. The Leap Motion Controller is a small, accurate and affordable consumer device, which is able to detect and track objects without the need of affixing sensors to them [20].

The main objective of this work is to study the feasibility of tracking laparoscopic instruments using the Leap Motion Controller inside a box trainer, which, to the authors' knowledge, has never been studied before. A series of experiments are presented in order to determine its static and dynamic precision. This study is the first step in creating an application in which the motion of the tip of the instrument can be tracked during laparoscopic training by using the Leap Motion Controller.

\section{Materials and methods}

\subsection{Laparoscopic instruments}

Laparoscopic instruments are designed to allow access into the patient's body through small incisions. Typical design consists of an elongated, monochromatic (usually in black color) rigid shaft with a diameter of $5 \mathrm{~mm}$, connecting a distal and a proximal end (Fig. 1). The distal end consists of a pair of metallic jaws that can be opened and closed, and which depending on their shape, allow the surgeons to perform actions such as dissection or grasping. The proximal end provides the instrument handle, which allows control of the jaws in four degrees of freedom (left/right pivoting, up/down pivoting, insertion/ withdrawal, clockwise/anti-clockwise rotation) [21].

The surgical scenario (whether a real patient or a box trainer) is constrained by the view provided by the endoscope. In the case of the laparoscopic instruments, this means that only the distal end and part of the shaft will be visible on screen.

\subsection{The Leap Motion Controller}

The Leap Motion Controller uses two optical sensors and three infrared LEDs to detect objects within its field of view, which is located above the black surface of the controller [20]. It is able to recognize long thin objects, giving the location of their tip in Cartesian coordinates for every single frame.

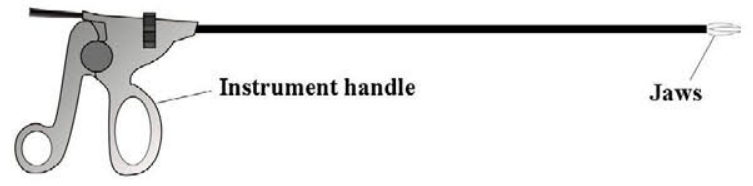

Fig. 1. Laparoscopic instrument model. 
Potential advantages of using Leap Motion for tracking laparoscopic instruments inside a box trainer mainly focus on its size, ease of use and portability. The device has a size of $8 \times 3 \times 1.2 \mathrm{~cm}$ and a weight of approximately $45 \mathrm{~g}$, and can be easily attached to any surface inside the box trainer, where direct line of sight is always kept with the laparoscopic instruments. Since no additional sensors need to be mounted in the instruments, their ergonomic properties are not altered at any time. This is a feature shared with computer vision-based alternatives such as the EVA Tracking System, with the additional advantage of being commercially available and backed up by a community of developers and app market [20].

As is the case of computer vision alternatives, the lighting and setting influence the robustness of tracking. The frame rate is approximately 80 frames per second in normal mode and 40 frames per second in robust mode, which cannot be changed manually. Robust mode is automatically entered when the device is used in bright lighting conditions or non-optical lightning scenarios, with a slight penalty to accuracy and range. The origin of the coordinate system is located at the center of the top surface of the Leap Motion Controller. The $X$-axis lies parallel with respect to the long edge of the device, whereas the $Z$-axis runs orthogonal to this edge. The $Y$-axis runs perpendicular to the black surface of the device (Fig. 2).

Due to its recent introduction in the market, there are currently only a few studies present in the literature featuring the Leap Motion Controller [22-27]. The first four studies focus on applications using hand gestures. In particular, [24] and [25] address the use of Leap Motion inside the OR to control medical imaging equipment and teleassisted robotic systems respectively. Both studies provide proof of the Leap Motion validity to recognize hand gestures in the OR. However, neither study focuses on its use for instrument tracking purposes, and therefore, they deviate from the purpose of this research.

The latter two studies focus on the performance of the Leap Motion Controller itself. The study by Weichert et al. [26] provides an analysis of the accuracy and robustness of the Leap Motion Controller. An average accuracy of $0.7 \mathrm{~mm}$ was determined, which is higher than that obtained from comparable controllers in the same price range (e.g. Microsoft Kinect ${ }^{\mathrm{TM}}$ ). The study by Guna et al. [27] is an analysis of the precision and repeatability of the Leap Motion Controller and its suitability for static and dynamic tracking using different tracking objects and configurations. The authors stated that there is a significant drop in accuracy for samples taken more than $250 \mathrm{~mm}$ away from the controller. It must however be

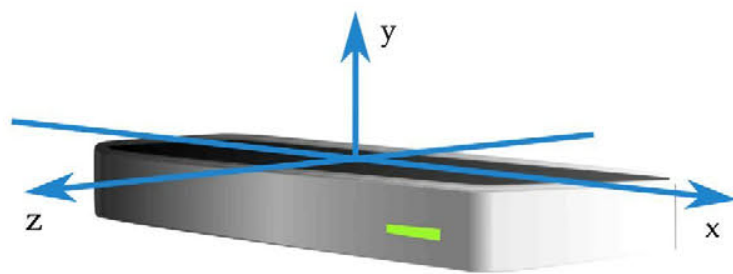

Fig. 2. Cartesian coordinate system of the Leap Motion Controller. noted that when tracking laparoscopic instruments using Leap Motion Controller inside a box trainer, the tip of the instrument should typically not exceed the $250 \mathrm{~mm}$ distance with respect to the sensor.

\subsection{Experimental setting and evaluation platform}

A Leap Motion Controller was used for all experiments. During the experiments the device was either fixed to a horizontal surface (experiments outside the box trainer), or unobtrusively attached to a vertical wall (experiments inside the box trainer, Fig. 3).

A laparoscopic dissector was used (Covidien Autosuture Endo Dissect $5 \mathrm{~mm}$, Covidien, Dublin, Ireland) for all experiments. For the static control experiments outside the box trainer, the laparoscopic instrument was held horizontally above the Leap Motion Controller by a cardboard structure. For the experiments inside the box trainer, an evaluation platform was designed and created to hold the laparoscopic instrument in a fixed position. Fig. 3 shows this platform as well as the experimental set-up inside the box trainer. The platform, consisting of two plateaus, was created from wood. Holes were drilled, resulting in $3 \times 4$ holes equally distributed with a mutual distance of approximately $25 \mathrm{~mm}$. The holes of the upper plateau have a diameter of $8 \mathrm{~mm}$, which corresponds with the outer diameter of a laparoscopic trocar. The holes of the lower plateau have a diameter of $8 \mathrm{~mm}$ for the top $25 \mathrm{~mm}$ and a diameter of $5 \mathrm{~mm}$ down to the bottom of the plateau, which corresponds with the diameter of the shaft of the instrument. This configuration allowed fixing the trocar and instrument position steadily within the platform for static experiments. It also allowed for vertical movements of the laparoscopic instrument during dynamic experiments.

The platform was placed and fixed on top of a box trainer currently being used and validated for laparoscopic training (SIMULAP ${ }^{\circledR}$, Jesús Usón Minimally Invasive Surgery Centre, Cáceres, Spain) [28]. In this manner, it was possible to hold still the laparoscopic instrument during the experiments inside the box trainer. The distance between the Leap Motion Controller and the first 3 positions of the evaluation platform was approximately $110 \mathrm{~mm}$ ( $Y$-axis), whereas with respect to the last 3 positions it was approximately $185 \mathrm{~mm}$.

Pre-experimental trials indicated that a few modifications would be needed to be able to track the laparoscopic instrument. The first modification was to place a bright marker around the distal end of the black shaft of the instrument, to overcome the fact that the Leap Motion Controller is not able to track dark colors. Three different colors were tested: yellow, green and red. The markers, made from heat shrink tubing, have the same diameter as the shaft of the instrument and are $75 \mathrm{~mm}$ long. The second modification was to darken the surface behind the instrument inside the box trainer, as the Leap Motion Controller was incapable of recognizing the instrument with a clear background behind. A configuration of 9 LED lights was used to maintain enough light to provide visibility inside the box trainer (Fig. 3).

Specific software was developed in $\mathrm{C}++$ using the Leap Motion Tracking SDK to track the coordinates of the 


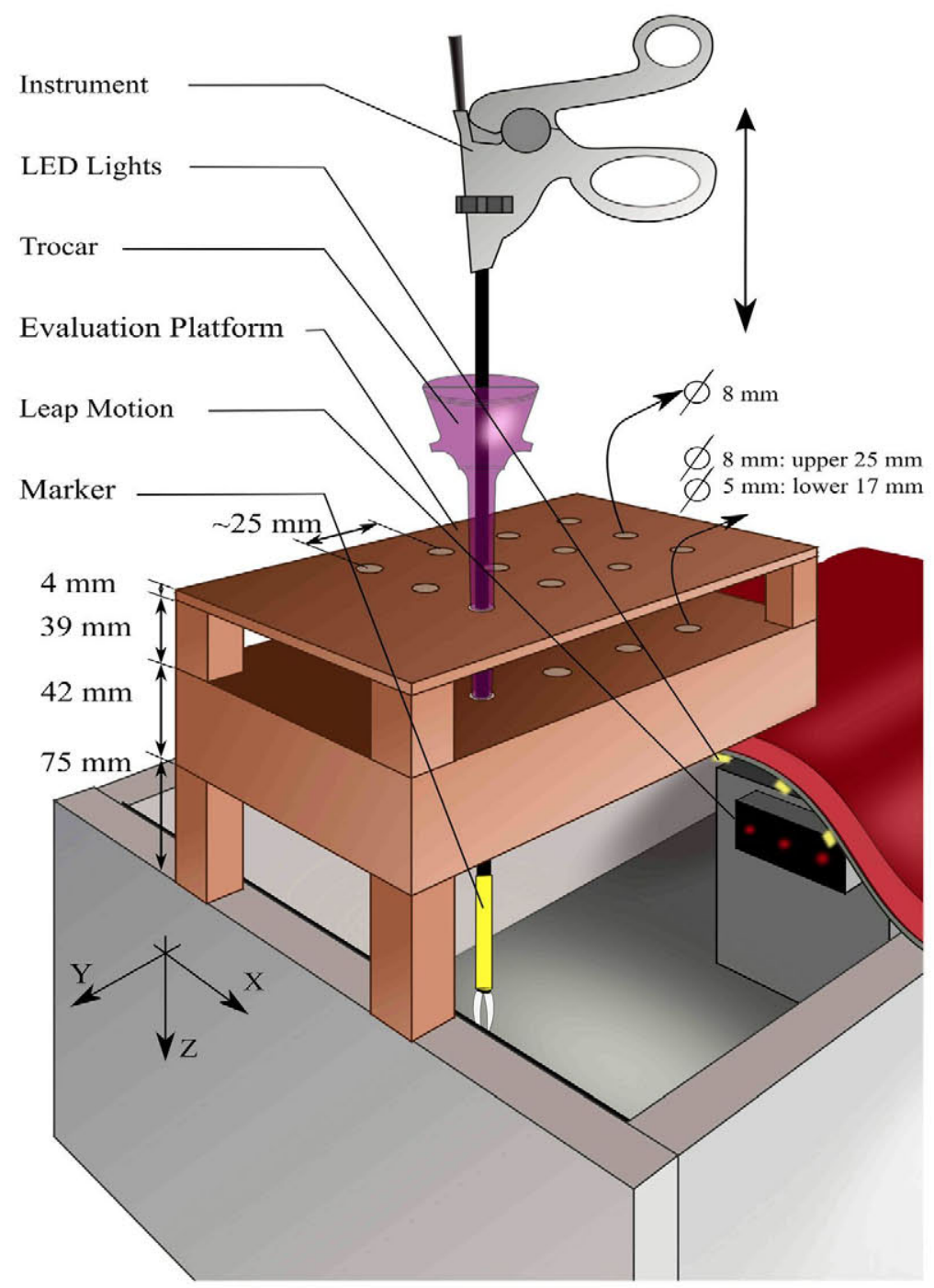

Fig. 3. Experimental set-up. The evaluation platform was used for the experiments inside the laparoscopic box trainer. It has 12 equally distributed holes and is able to hold the laparoscopic instrument in a fixed position.

instrument (measured at the middle of the markers' distal end [29]). The data were saved for each sample as a comma-separated value file (CSV) containing the $X, Y$, and $Z$ coordinates per frame. A CSV file was chosen, as it could be easily opened by MATLAB ${ }^{\mathbb{1}}$ (The MathWorks, Inc., Natick, MA, USA), enabling data analysis after the experiments were performed.

\subsection{Experiments}

Following a similar structure of experiments proposed by Guna et al. [27], 4 experiments ( 3 static and 1 dynamic) were carried out to determine the precision of the Leap Motion Controller for tracking laparoscopic instruments (Table 1). Static experiments were designed to measure the dispersion of the Leap Motion as a means to characterize the repeatability of measurements. The first two experiments examined long and short term precision and the resolution of the Leap Motion Controller at a given position, to determine its temporal dispersion. They were carried out both inside and outside the box trainer, to analyze differences between both settings. The third experiment was designed to provide information on the distortion of the Leap Motion's perception of space across the whole working volume inside the box trainer and provide data on its spatial dispersion. Finally, the dynamic experiment was designed to measure the distortion of the Leap Motion's perception of distance traveled for all available positions along the depth of the platform.

All experiments were done using the green, yellow and red markers, to allow for color comparison. For the experiments inside the box trainer, the evaluation platform was used. The method of the individual experiments will be explained in more detail in the following sub-sections.

\subsubsection{Static long precision experiment}

This experiment was performed inside and outside the box trainer for each of the three markers. The laparoscopic 
Table 1

Experiments and specifications. The total number of measurements is calculated by multiplication of 3 (number of markers)*\#positions*\#measurements/marker $* 2$ (if experiment is conducted inside and outside of the box trainer)

\begin{tabular}{|c|c|c|c|c|c|c|c|c|}
\hline Exp. & $\begin{array}{l}\text { Static }(S) / \\
\text { Dynamic } \\
\text { (D) }\end{array}$ & Goal & Time & $\begin{array}{l}\text { Traveled } \\
\text { distance }\end{array}$ & $\begin{array}{l}\# \\
\text { Positions }\end{array}$ & $\begin{array}{l}\text { \# } \\
\text { Measurements } \\
\text { /marker }\end{array}$ & $\begin{array}{l}\text { Inside (I)/ } \\
\text { Outside } \\
\text { (O) }\end{array}$ & $\begin{array}{l}\text { \# Total } \\
\text { measurements }\end{array}$ \\
\hline 1 & $\mathrm{~S}$ & Determine long term precision & $2 \mathrm{~h}$ & - & 1 & 1 & $I \& O$ & 6 \\
\hline 2 & $\mathrm{~S}$ & Determine short term precision & $15 \mathrm{~s}$ & - & 1 & 20 & $I \& O$ & 120 \\
\hline 3 & $\mathrm{~S}$ & Determine static resolution & $15 \mathrm{~s}$ & - & $\begin{array}{l}12 \\
\text { (platform) }\end{array}$ & 10 & $\mathrm{I}$ & 360 \\
\hline 4 & $\mathrm{D}$ & $\begin{array}{l}\text { Determine ability to calculate the path } \\
\text { length traveled by the tip of the instrument }\end{array}$ & - & $200 \mathrm{~mm}$ & $\begin{array}{l}12 \\
\text { (platform) }\end{array}$ & 10 & $\mathrm{I}$ & 360 \\
\hline
\end{tabular}

instrument was held for two hours in the same position, with the Leap Motion Controller continuously tracking it, to examine the long term precision. Data were analyzed using MATLAB, calculating the mean and standard deviation for each measurement.

\subsubsection{Static short precision experiment}

This experiment was performed inside and outside the box trainer for each of the three markers. The instrument was held in the same position during the experiment. The Leap Motion Controller tracked the instrument 20 times for $15 \mathrm{~s}$, to test the short term precision, resulting in a total of 120 measurements. The instrument was not moved or replaced in between measurements. Data were analyzed using MATLAB calculating the deviation from the mean for each measurement.

\subsubsection{Static distance experiment}

This experiment was performed inside the box trainer for each of the three markers. The goal of the experiment was to establish the precision of the Leap Motion Controller in the determination of distances when the instrument is held still in different positions. The instrument was placed at each position of the evaluation platform and was tracked with the Leap Motion Controller for $15 \mathrm{~s}$. This was repeated 10 times for every position on the platform, resulting in a total of 360 measurements.

Static distances between two adjacent points in 3D space were calculated by using the Euclidean Distance Formula:

Static Distance $=\sqrt{\left(x_{p}-x_{a p}\right)^{2}+\left(y_{p}-y_{a p}\right)^{2}+\left(z_{p}-z_{a p}\right)^{2}}$

With $x_{p}, y_{p}$, and $z_{p}$ being the mean of the coordinates corresponding to the tracked point at one platform position and $x_{a p}, y_{a p}$, and $z_{a p}$ the mean of the coordinates belonging to the same point at the adjacent position. Mean distances and standard deviations were calculated and analyzed using MATLAB.

In order to determine if the precision of the Leap Motion Controller decreased the further away the laparoscopic instrument, a multiple-sample test for equal variances in MATLAB was used to check whether the calculated distances presented no significant differences between variances. A p-value lower than 0.05 confirms this null-hypothesis.

\subsubsection{Dynamic experiment}

This experiment was performed inside the box trainer for each of the three markers. The goal of the experiment was to determine the Leap Motion precision when moved a certain, fixed distance. The instrument was placed at each position of the evaluation platform and slowly moved up and down manually 2 times for $50 \mathrm{~mm}$, making a total path length of $200 \mathrm{~mm}$. Two small markers placed on the instrument shaft $50 \mathrm{~mm}$ apart indicated the start and ending positions of the instrument within the platform to guide the movements. The measurement was repeated 10 times for every position on the platform, resulting in a total of 360 measurements.

Dynamic distances between points in 3D space for successive frames were calculated. To do so, the Euclidean Distance Formula was used:

$$
\text { Dynamic Distance }=\sqrt{\left(x_{i}-x_{i-1}\right)^{2}+\left(y_{i}-y_{i-1}\right)^{2}+\left(z_{i}-z_{i-1}\right)^{2}}
$$

With $x_{i}, y_{i}$, and $z_{i}$ being the coordinates of the tracked point of the current frame and $x_{i-1}, y_{i-1}$, and $z_{i-1}$ the coordinates belonging to the same point of the previous frame. For each position, the mean and standard deviation of the distance traveled over the 10 repetitions was obtained. In order to ascertain the influence of manual movements, Pearson's correlation between the mean distance and the average speed of movements was analyzed, for each position and marker. Values $>0.7$ would be considered to show a strong dependency between the path measured by the Leap Motion and the speed of movements.

\section{Results and discussion}

\subsection{Static long precision experiment}

Static long precision results are presented in Fig. 4. Results are presented as deviation from the mean, with the deviation of the Cartesian $x, y$ and $z$ coordinates in $\mathrm{mm}$ on the $Y$-axis and the captured frame on the $X$-axis. Due to the lighting conditions of the environment, the Leap Motion Controller entered automatically robust mode for the experiments outside the box trainer, resulting in more than 275,000 frames per measurement. Inside the box trainer more than 550,000 frames per measurement were obtained (representing $2 \mathrm{~h}$ ).

The outside measurements had in general a lower deviation from the mean compared with the inside 

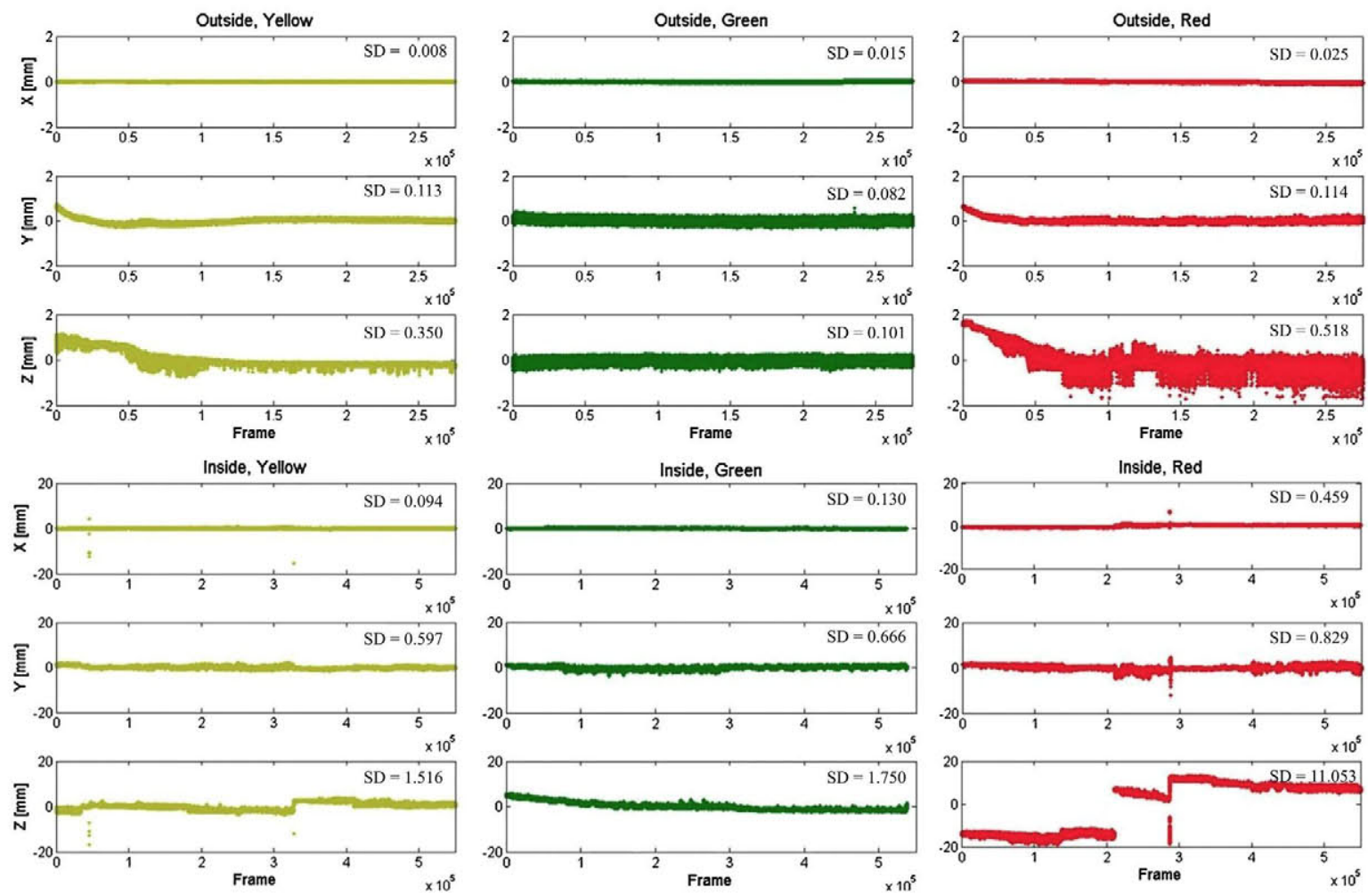

Fig. 4. Static Long Precision Experiment. The deviation from the mean for all frames of the $x, y$ and $z$ coordinates are shown for the yellow (column \#1), green (column \#2) and red (column \#3) marker, both inside and outside the box trainer. Experiment time was two hours, resulting in $>275,000$ frames for the outside situation (robust mode) and $>550,000$ frames for the inside situation. (For interpretation of the references to color in this figure legend, the reader is referred to the web version of this article.)

measurements. For all markers, the range of deviation was smallest for the $x$-coordinate and largest for the $z$-coordinate. The results obtained with the green marker were continuous both outside and inside the box, whereas the yellow and red marker showed some discontinuities for the experiments inside the box. All standard deviations were smaller than $2 \mathrm{~mm}$, except for the results of the $z$-coordinate for the experiment with the red marker $(\mathrm{SD}=11.05 \mathrm{~mm})$. The smallest standard deviation was obtained for the deviation of the $x$-coordinate with the yellow marker outside the box trainer $(\mathrm{SD}=0.008 \mathrm{~mm})$.

\subsection{Static short precision experiment}

The results of the static short precision experiment are illustrated in Fig. 5 as a scatterplot. The $x, y$ and $z$ deviations are presented on the $Y$-axis, whereas the $X$-axis represents the type of the experiment (inside or outside the box trainer for every marker color).

All deviations were smaller than $2.5 \mathrm{~mm}$, with the smallest deviations occurring for the $x$-coordinate. The deviation from the mean was smaller for the experiments outside the box trainer than inside the box trainer, except for the $z$-coordinate of the red marker. In general, the largest spread was found for the red marker.

The results of the static long and short term precision experiments showed that the Leap Motion Controller performed better outside than inside the box trainer, as standard deviations and deviations from the mean are lower. This could be explained by the fact that inside the box trainer there was a surface close behind the laparoscopic instrument and by the fact that the lightning conditions inside the box were different than outside. In general, green and yellow markers presented the best results for these experiments. The red marker was found to be less precise with respect to the other two colors: the first experiment showed larger discontinuities and the second experiment a larger spread in terms of deviation from the mean.

\subsection{Static distance experiment}

Results of the static distance experiment are shown in Fig. 6. The 12 positions of the evaluation platform are illustrated, as well as the relative position of the Leap Motion Controller with respect to them. The results are shown as the mean and standard deviation for the 10 repetitions of the calculated distances between two adjacent positions. Within this statistical analysis, 3 measurements were excluded from the data set, according to the guidelines given in [30]: two outliers were found at position 3 for the green and red marker and one outlier was found at position 10 for the green marker. These outliers deviated approximately 3 times the standard deviation of the mean of the corresponding $z$-coordinate.

The average standard deviation for all positions was $0.27 \mathrm{~mm}, 0.3 \mathrm{~mm}$ and $0.34 \mathrm{~mm}$ for the yellow, green and 

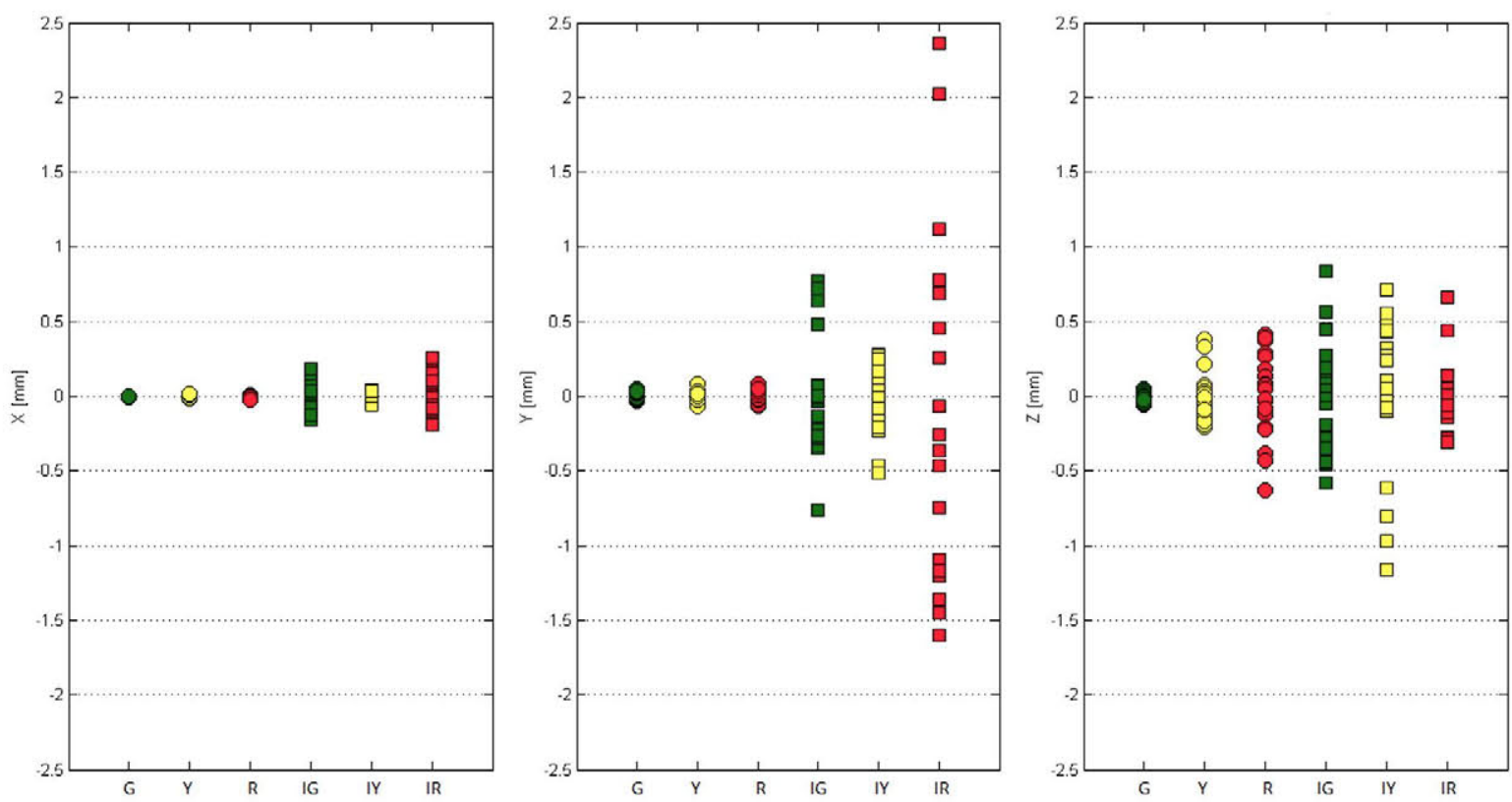

Fig. 5. Static Short Precision Experiment. Results displayed as deviation from the mean of the tip of the instrument (20 times for $15 \mathrm{~s}$ ). Legend: $\mathrm{G}=\mathrm{Green}$, $\mathrm{Y}=$ Yellow, $\mathrm{R}=$ Red, $\mathrm{I}=$ Inside Box, $\square=$ Inside, $\mathrm{O}=$ Outside. (For interpretation of the references to color in this figure legend, the reader is referred to the web version of this article.)

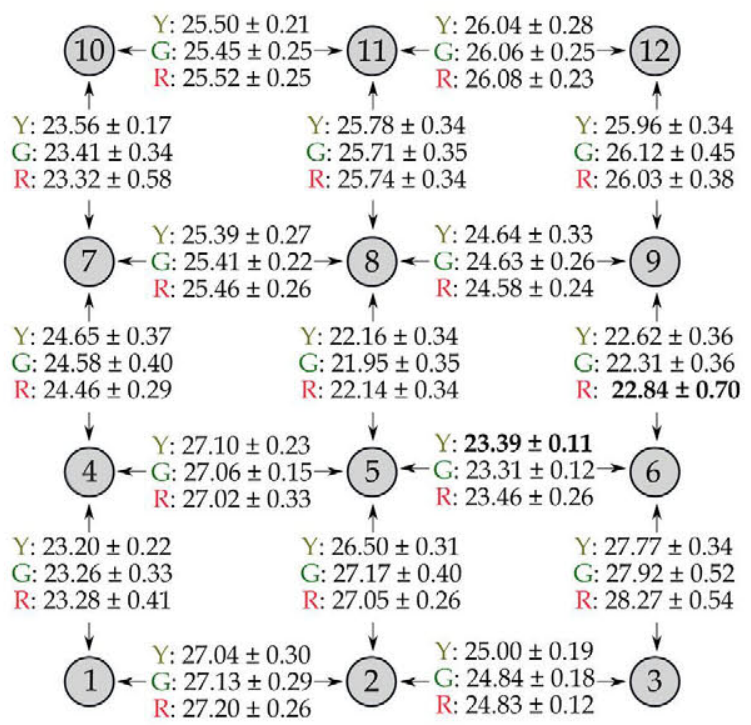

\section{Leap Motion}

Fig. 6. Static Distance Experiment. The mean and standard deviation are shown in $\mathrm{mm}$ for all calculated distances between adjacent positions of the evaluation platform, and for all markers. Boldface: highest (positions 6-9) and lowest (positions 5-6) deviations.

red markers respectively. In general, the standard deviations belonging to the calculated distances were equal to or lower than $0.7 \mathrm{~mm}$. Only in two specific instances, for the red marker, did the standard deviation reach above $0.5 \mathrm{~mm}$, again possibly due to the difficulties in tracking this specific color. This was consistent with the results reported by Guna et al. which set static standard deviations of the Leap Motion below $0.5 \mathrm{~mm}$ [27].

The multiple-sample test for equal variances gave a $p$ value of $0.59(>0.05)$. This implied that no significant differences could be found in variance for distances closer to or further away from the Leap Motion Controller. This result was also in accordance with the functioning range established by Guna et al. [27], and supported our earlier hypothesis that within the box trainer there will be no significant drops in precision consistency due to distance from the Leap Motion.

\subsection{Dynamic experiment}

The results of the dynamic experiment are shown in Fig. 7. The figure depicts the mean traveled distance and corresponding standard deviation for all markers and positions measured by the Leap Motion Controller. Almost all mean distances were bigger than $200 \mathrm{~mm}$. The smallest captured mean distance was found at position 3 for the red marker $(199 \mathrm{~mm})$, whereas the largest mean distance was found at position 8 , also for the red marker $(227 \mathrm{~mm})$.

In total, average standard deviation between platform positions of $4.25 \mathrm{~mm}, 7.08 \mathrm{~mm}$ and $7.67 \mathrm{~mm}$ were obtained for the yellow, green and red markers respectively when covering a distance of $200 \mathrm{~mm}$. Standard deviations were within a range of $2-15 \mathrm{~mm}$; with a standard deviation of $15 \mathrm{~mm}$ at positions 1 and 2 for the red and green marker respectively and a standard deviation of $2 \mathrm{~mm}$ at positions 5 and 9 for the red marker and position 
(10) $Y: 212 \pm 5(25.5)$
G: $218 \pm 4(23.1)$
R: $223 \pm 10(22.9)$

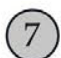

Y: $208 \pm 4(20.9)$

G: $215 \pm 4$ (20.6)

R: $214 \pm 4$ (21.8)
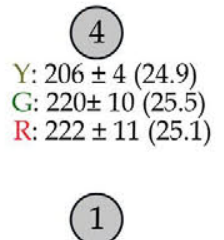

Y: $215 \pm 6(17.7)$

G: $218 \pm 13(16.1)$

R: $210 \pm 15$ (17.2)
(11)

$\mathrm{Y}: 212 \pm 3(25.3)$

G: $220 \pm 6(26.1)$

R: $224 \pm 11$ (23.4)

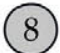

Y: $214 \pm 4(21.6)$

G: $219 \pm 5(23.7)$

R: $227 \pm 6(20.5)$

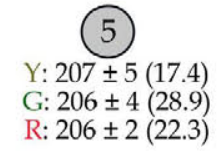

(2)

$Y: 212 \pm 2(16.5)$

G: $208 \pm 15(20.9)$

R: $219 \pm 9(16.6)$

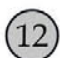

Y: $220 \pm 6(27)$

G: $223 \pm 4(30.5)$

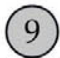

$Y: 212 \pm 6$ (19.2)

G: $218 \pm 4$ (22)

R: $210 \pm 2$ (21.5)

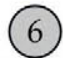

$\mathrm{Y}: 221 \pm 3(21)$

G: $216 \pm 4(19.5)$

R: $216 \pm 6$ (18.3)

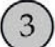

Y: $215 \pm 3(15.7)$

R: $199 \pm 11$ (18)
R: $218 \pm 5$ (27.9)

G: $206 \pm 12$ (15.6)

\section{Leap Motion}

Fig. 7. Dynamic Experiment. The mean and standard deviation of the calculated path length are shown in $\mathrm{mm}$ for all adjacent positions of the evaluation platform and markers (Yellow, Green and Red). In brackets: Average speed of movement over 10 repetitions for each position and marker $(\mathrm{mm} / \mathrm{s})$. (For interpretation of the references to color in this figure legend, the reader is referred to the web version of this article.)

2 for the yellow marker. Most frequent standard deviation was of $4 \mathrm{~mm}$, with 10 occurrences. Results obtained with the yellow marker had on average lower standard deviations than those obtained with the green and red markers.

Guna et al. [27] reported a drop in consistency for dynamic experiments, especially at long distances. They doubted that in its current version the device could be used for professional tracking. However, it has been shown that in order to perform motion analysis of surgeons' movements inside a box trainer, these ranges of performance can be acceptable [19].

A possible limitation involved the manual movement of the laparoscopic instrument during the experiment. While the start and end markers provided a suitable reference, manual control made the existence of small variations between repetitions a possibility. Movements were carefully guided through the evaluation platform to keep a steady velocity, and Pearson's correlation values between mean distance travelled and average speed were of 0.09 , 0.323 and 0.358 for the yellow, green and red markers respectively. These results suggested that there was no strong correlation between the results obtained and the speed of manual movements during the test.

\subsection{Study limitations and future work}

The main purpose behind this study has been to determine the feasibility of the Leap Motion Controller for tracking laparoscopic instruments inside a box trainer setting. As such, we have limited the complexities of the training scenario to the minimum requirements, focused on determining the working conditions of the Leap Motion Controller inside the box trainer (background, markers). The scenario has lacked the inclusion of more complex elements, such as surgical tasks, ex-vivo organs or multiple instruments. Likewise, only one type of instrument (laparoscopic dissector) was employed. Considering that tracking is based primarily on information from the marker, we hypothesize that tracking of other instruments should be possible. Finally, dynamic experiments were kept simple trying to cover as much as possible the working space in the box trainer.

In this regard, future work should move toward a more realistic setting increasing the complexity of the box trainer. Surgical tasks and ex-vivo organs used for training and assessment should be included. Additionally, the ability of the Leap Motion Controller to track two instruments simultaneously should be tested. The Leap Motion Controller itself is able to track multiple objects and, therefore, it is hypothesized that calculating the path length for two instruments will be feasible using different color markers to distinguish between them. Further experiments considering random movements of the instruments and a finer control of the instrument should be performed to confirm whether the Leap Motion Controller can be consistent and predictable in detecting and tracking instruments inside a box trainer. Finally, all of these elements should be combined and tested with real surgeons (novels, residents and experts) to perform motion analysis and validate the use of Leap Motion to assess basic surgical skills.

Future studies should also aim to determine the accuracy of the Leap Motion Controller, and compare it with other available tracking systems. Electromagnetic systems such as Aurora (Northern Digital Inc.; ON, Canada) or microBIRD (Ascension, VT, USA) have a reported accuracy of $0.96 \mathrm{~mm} \pm 0.68 \mathrm{~mm}$ and $1.14 \pm 0.78 \mathrm{~mm}$ for distances below $50 \mathrm{~mm}$. Optical tracking technologies such as Polaris (Northern Digital Inc.; ON, Canada) provide accuracy results under $0.25 \mathrm{~mm}$ for a $240 \times 156 \times 131 \mathrm{~cm}$ of field of measurement [31]. However, these systems tend to be expensive and require the use of sensors/fiducials mounted on the laparoscopic instruments. Computer-vision alternatives offering non-obtrusive, portable tracking such as the EVA Tracking System, report accuracies of $1.2 \mathrm{~cm}$ [32].

A priori, these accuracy values are comparable to those reported by Weichert et al. for the Leap Motion Controller [26], which place it on $0.7 \mathrm{~mm}$ for the Leap Motion when tracking a pen-like object. However, it would be necessary to test them under the specific conditions of a training setting. In order to do that, a reference system would be required as a ground truth for the experiment. In this sense, any of the aforementioned systems could be employed. New experiments to determine both accuracy and precision with the laparoscopic instrument at different angles could be carried out, as in this study the instrument was held vertically with respect to the controller. We expect that the angle of the instrument will not be a problem, as the study by Guna et al [27] did not find a drop in consistency of the Controller when the inclination of tracking objects had been changed. This should, however, be verified when the Leap Motion Controller is used inside a box trainer. 


\section{Conclusions}

This study shows the potential of the Leap Motion Controller for tracking laparoscopic instruments within a box trainer. As a preliminary research work, the study only aims to verify the feasibility of using the Leap Motion Controller as an alternative for tracking laparoscopic instruments and provide evidence of its usefulness as a ground for further research. Reported precision values reflect an acceptable performance in its current version, although further proof is required in more demanding conditions (e.g.: multiple instrument tracking). Tracking of the instruments requires the use of color markers, and in particular yellow and green seem valid options, although further colors should be tested. The findings presented could be used to develop an application that is able to follow the movements of the laparoscopic instruments in a box trainer during a training task using the Leap Motion Controller. In this way, the Leap Motion Controller could be analyzed as a viable alternative to objectively measure the skills of residents during training. However, further studies should still be carried out to verify the accuracy level of the system.

The Leap Motion Controller presents a series of advantages that make it suitable to perform motion analysis in training scenarios: (1) its portability and ease of use, which make it easily adaptable to different models of box trainers; (2) its capability of tracking the laparoscopic instruments in a transparent and unobtrusive manner, without the need of mounting sensors that may modify the ergonomic properties of the laparoscopic instruments; and (3) its low cost $(89.99 €$ at the time of writing this report [33]) and widespread availability. A limitation of the system is that it can be only used in training settings (as opposed to real surgeries), as it requires placing the Controller within the surgical scenario. However, this limitation is shared by other systems built for the same purpose, bearing in mind that their scope covers applications in training settings [12,16-18].

Leap Motion constitutes an important addition to the family of tracking systems for laparoscopic instruments. Similarly to other sensor-based systems, it could become a useful tool to perform motion analysis of surgeons' performance in training tasks. Moreover, the advantages of the Leap Motion Controller can potentially appeal to the different stakeholders on the field at different levels: from hospitals/training centers looking to provide advanced tracking capabilities to their existing training settings, to individual surgeons who may use it to practice outside the hospital facilities (at home, for example), to IT/simulation companies in the business of developing virtual reality/augmented reality/serious games simulators for laparoscopic training and assessment.

\section{Acknowledgements}

This study has been carried out under the framework of Project NAVISurg, sponsored by the Networking Research Center on Bioengineering, Biomaterials and Nanomedicine (CIBER-BBN).

\section{References}

[1] J. Usón, F.M. Sánchez-Margallo, S. Pascual, S. Climent, Formación En Cirugía Laparoscópica Paso a Paso, $4^{\mathrm{s}}$ Ed., Centro de Cirugía de Mínima Invasión Jesús Usón, Cáceres, 2013.

[2] E.G. Chekan, T.N. Pappas, General principles of minimally invasive surgery, in: J. Norton, R.R. Bollinger, A. Chang, S. Lowry, S. Mulvihill, H. Pass, R. Thompson (Eds.), Surgery SE - 25, Springer, Berlin Heidelberg, 2001, pp. 429-453, http://dx.doi.org/10.1007/978-3642-57282-1_25.

[3] W.J. Lee, C.P. Chan, B.Y. Wang, Recent advances in laparoscopic surgery, Asian J. Endosc. Surg. 6 (1) (2013) 1-8.

[4] S. Cotin, N. Stylopoulos, Metrics for laparoscopic skills trainers: the weakest linkl, in: T. Dohi, R. Kikinis (Eds.), Medical Image Computing and Computer-Assisted Intervention - MICCAI 2002, Springer, Berlin Heidelberg, 2002, pp. 35-43.

[5] S. Tsuda, D. Scott, J. Doyle, D.B. Jones, Surgical skills training and simulation, Curr. Probl. Surg. 46 (4) (2009) 271-370.

[6] K. Roberts, R.L. Bell, A.]. Duffy, Evolution of surgical skills training, World J. Gastroenterol. 12 (20) (2006) 3219-3224.

[7] A. Supe, R. Prabhu, I. Harris, S. Downing, A. Tekian, Structured training on box trainers for first year surgical residents: does it improve retention of laparoscopic skills? A randomized controlled study, J. Surg Educ. 69 (5) (2012) 624-632, http://dx.doi.org/ 10.1016/j.jsurg.2012.05.002.

[8] D.J. Scott, P.C. Bergen, R.V. Rege, R. Laycock, S.T. Tesfay, R.J. Valentine, D.M. Euhus, D.R. Jeyarajah, W.M. Thompson, D.B. Jones, Laparoscopic training on bench models: better and more cost effective than operating room experience?, J Am. Coll. Surg. 191 (3) (2000) 272283, http://dx.doi.org/10.1016/S1072-7515(00)00339-2.

[9] 1. Oropesa, P. Sánchez-González, P. Lamata, M.K. Chmarra, J.B. Pagador, J.A. Sánchez-Margallo, F.M. Sánchez-Margallo, E.J. Gómez, Methods and tools for objective assessment of psychomotor skills in laparoscopic surgery, J. Surg. Res. 171 (1) (2011) e81-e95.

[10] S.A. Fraser, D.R. Klassen, L.S. Feldman, G.A. Ghitulescu, D. Stanbridge, G.M. Fried, Evaluating laparoscopic skills: setting the pass/fail score for the MISTELS system, Surg. Endosc. 17 (2003) 964-967, http://dx. doi.org/10.1007/s00464-002-8828-4.

[11] P.D. van Hove, G.J.M. Tuijthof, E.G.G. Verdaasdonk, L.P.S. Stassen, J. Dankelman, Objective assessment of technical surgical skills, Br. J. Surg. 97 (7) (2010) $972-987$.

[12] J. Rosen, M. Solazzo, B. Hannaford, M. Sinanan, Task decomposition of laparoscopic surgery for objective evaluation of surgical residents' learning curve using hidden markov model, Comput. Aided Surg. 7 (2002) 49-61.

[13] H.C. Lin, I. Shafran, D. Yuh, G.D. Hager, Towards automatic skill evaluation: detection and segmentation of robot-assisted surgical motions, Comput. Aided Surg. 11 (5) (2006) 220-230.

[14] N. Ahmidi, P. Poddar, J.D. Jones, S.S. Vedula, L. Ishii, G.D. Hager, M. Ishii, Automated objective surgical skill assessment in the operating room from unstructured tool motion in septoplasty, Int. J. Comput. Assist. Radiol. Surg. 10 (6) (2015) 981-991, http://dx.doi.org/ 10.1007/s11548-015-1194-1.

[15] A. Malpani, S.S. Vedula, C. Chiung, G. Chen, G.D. Hager, Pairwise comparison-based objective score for automated skill assessment of segments in a surgical task, in: Information Processing in ComputerAssisted Interventions, Lecture Notes in Computer Science, vol. 8498, 2014, pp. 138-147.

[16] M.K. Chmarra, N.H. Bakker, C.A. Grimbergen, J. Dankelman, TrEndo, a device for tracking minimally invasive surgical instruments in training setups, Sensor Actuat. A-Phys. 126 (2) (2006) 328-334.

[17] V. Datta, S. Mackay, M. Mandalia, A. Darzi, The use of electromagnetic motion tracking analysis to objectively measure open surgical skill in the laboratory-based model, J. Am. Coll. Surg. 193 (5) (2001) 479-485

[18] T.M. Peters, Image-guidance for surgical procedures, Phys. Med. Biol. 51 (14) (2006) R505-R540.

[19] I. Oropesa, P. Sánchez-González, M.K. Chmarra, P. Lamata, A. Fernández, J.A. Sánchez-Margallo, F.W. Jansen, J. Dankelman, F.M. Sánchez-Margallo, E.J. Gómez, EVA: Laparoscopic instrument tracking based on endoscopic video analysis for psychomotor skills assessment, Surg. Endosc. 27 (3) (2013) 1029-1039.

[20] Leap Motion, Documentation developers' guide - API overview, 2014 $<$ https://developer.leapmotion.com> (Retrieved 08.04.14).

[21] B. Iacovelli, Laparoscopic Instruments, United States of America, 1994,5350391

[22] H. Regenbrecht, J. Collins, S. Hoermann, A leap-supported, hybrid AR interface approach, in: Proceedings of the 25th Australian Computer-Human Interaction Conference, Augmentation, 
Application, Innovation, Collaboration, 2013, ACM, 2013, pp 81-284.

[23] S. Vikram, L. Li, S. Russell, Writing and sketching in the air recognizing and controlling on the fly, in: CHI ' 13 Extended Abstracts on Human Factors in Computing Systems, 2013, ACM, pp. $1179-1184$.

[24] N. Bizzotto, A. Costanzo, L. Bizzotto, D. Regis, A. Sandri, B. Magnan, Leap motion gesture control with OsiriX in the operating room to control imaging: first experiences during live surgery, Surg. Innov. 21 (6) (2014) 655-656, http://dx.doi.org/10.1177/1553350614528384.

[25] F. Despinoy, S. Alonso, N. Zemiti, P. Jannin, P. Poignet, Comparative assessment of a novel optical human-machine interface for laparoscopic telesurgery, in: Information Processing in ComputerAssisted Interventions, Lecture Notes in Computer Science, vol. 8498, 2014, pp. 21-30).

[26] F. Weichert, D. Bachmann, B. Rudak, D. Fisseler, Analysis of the accuracy and robustness of the leap motion controller, Sensors 13 (5) (2013) 6380-6393, http://dx.doi.org/10.3390/s130506380.

[27] J. Guna, G. Jakus, M. Pogačnik, S. Tomažič, J. Sodnik, An analysis of the precision and reliability of the leap motion sensor and its suitability for static and dynamic tracking, Sensors 14 (2) (2014) 3702-3720, http://dx.doi.org/10.3390/s140203702.
[28] S. Enciso, F.M. Sánchez-Margallo, I. Díaz-Güemes, J. Usón, Preliminary validation of the Simulap $\left({ }^{\circledast}\right)$ physical simulator and its assessment system for laparoscopic surgery, Cir. Esp. 90 (1) (2012) $38-44$.

[29] Tool - Leap Motion C++ SDK v2.2 Documentation, <https:/ developer.leapmotion.com/documentation/java/api/Leap.Tool.html> (access April 2015).

[30] J.W. Osborn, A. Overbay, The power of outliers (and why researchers should always check for them), Pract. Assess. Res. Eval. 9 (6) (2004).

[31] J.A. Sánchez-Margallo, F.M. Sánchez-Margallo, J.B. Pagador, I. Oropesa, M.L. Hernández, E.J. Gómez, J.M. del Pozo, Technical evaluation of a third generation optical pose tracker for motion analysis and image-guided surgery, in: Clinical Image-Based Procedures. From Planning to Intervention. Lecture Notes in Computer Science, vol. 7761, 2013, pp. 75-82.

[32] I. Oropesa, J.A. Sánchez Margallo, P. Sánchez González, F.M. SánchezMargallo, E.J. Gómez, Comparative study of two video-based laparoscopic instrument tracking algorithms for image guided surgical applications, in: Proceedings of the II Joint Workshop on New Technologies for Computer/Robot Assisted Surgery, 2012.

[33] Leap Motion, 2014 <http://store-eur.leapmotion.com/> (access April 2015). 